\title{
Euler's divergent series and an elementary model in Statistical Physics
}

\author{
Bill Allombert \\ Institut de Mathématiques de Bordeaux, UMR 5251, Université de Bordeaux, \\ 351 cours de la Libération, F-33405 Talence Cedex, France \\ Jean-Paul Allouche \\ CNRS, Institut de Mathématiques de Jussieu-PRG, Université Pierre et Marie Curie, \\ Case 247, 4 Place Jussieu, F-75252 Paris Cedex 05, France \\ Michel Mendès France \\ Institut de Mathématiques de Bordeaux UMR 5251, Université de Bordeaux, \\ 351 cours de la Libération, F-33405 Talence Cedex, France
}

Received 3 April 2015, accepted 3 April 2016, published online 2 May 2016

\begin{abstract}
We discuss the multiple integral of a multivariate exponential taken with respect either to the Lebesgue measure or to the discrete uniform Bernoulli measure. In the first case the integral is linked to Euler's everywhere divergent power series and its generalizations, while in the second case the integral is linked to a one-dimensional model of spin systems as encountered in physics.

Keywords: Euler divergent series, Abel-Plana Formula, Stirling numbers, spin system, Ising chain. Math. Subj. Class.: 33E20, 28A35, 82B44, 82D30
\end{abstract}

\section{Introduction}

Consider the integral $(N \geq 1)$

$$
Z_{N}(x)=\int_{\mathbb{R}^{N}} \exp \left(-H\left(\sum_{j=1}^{N} u_{j}\right)-x \prod_{j=1}^{N} u_{j}\right) \mu\left(\prod_{j=1}^{N} d u_{j}\right)
$$

E-mail addresses: bill.allombert@math.u-bordeaux.fr (Bill Allombert), jean-paul.allouche@imj-prg.fr (Jean-Paul Allouche), michel.mendes-france@math.u-bordeaux.fr (Michel Mendès France)

(a) (i) This work is licensed under http://creativecommons.org/licenses/by/3.0/ 
If $\mu$ is the Lebesgue measure on $(0, \infty)^{N}$ and $H=1$, the integral is linked to the series

$$
\sum_{n \geq 0}(-1)^{n}(n !)^{N-1} x^{n}
$$

which, for $N=2$, is attributed to Euler. If $N=1$ the series reduces to $(1+x)^{-1}$ (convergent for $|x|<1$ ) and for $N \geq 2$ it diverges for all $x \neq 0$.

If on the other hand, $\mu$ is the Bernoulli measure on the set $\{-1,1\}^{N}$ then the integral reads

$$
Z_{N}(x)=\frac{1}{2^{N}} \sum_{\underline{u} \in\{ \pm 1\}^{N}} \exp \left(-H\left(\sum_{j=1}^{N} u_{j}\right)-x \prod_{j=1}^{N} u_{j}\right)
$$

and could represent a certain spin system described in Section 6.

\section{Euler's divergent series}

If $\mu$ is the Lebesgue measure on $(0, \infty)^{N}$, we suppose that $H>0$ and $x \geq 0$. There is no loss of generality in the choice $H=1$ in Formula 1.1; take new variables $v_{j}=H u_{j}$. Integrate

$$
Z_{N}(x)=\int_{(0, \infty)^{N}} \exp \left(-\left(\sum_{j=1}^{N} u_{j}\right)-x \prod_{j=1}^{N} u_{j}\right) \prod_{j=1}^{N} d u_{j}
$$

with respect to $d u_{N}$ to obtain

$$
Z_{N}(x)=\int_{(0, \infty)^{N-1}} \frac{\exp \left(-\left(\sum_{j=1}^{N-1} u_{j}\right)\right)}{1+x \prod_{j=1}^{N-1} u_{j}} \prod_{j=1}^{N-1} d u_{j} .
$$

Suppose $N \geq 2$ since the case $N=1$ is trivial. $Z_{N}$ converges for all complex $x$ outside the negative real axis $(-\infty, 0)$. Expand $\left(1+x \prod_{j=1}^{N-1} u_{j}\right)^{-1}$ into a formal power series

$$
Z_{N}(x)=\int_{(0, \infty)^{N-1}} \exp \left(-\sum_{j=1}^{N-1} u_{j}\right) \sum_{n \geq 0}(-1)^{n} x^{n} \prod_{j=1}^{N-1} u_{j}^{n} \prod_{j=1}^{N-1} d u_{j}
$$

If we accept to permute the summation with the integral, then

$$
Z_{N}(x)=\sum_{n \geq 0}(-1)^{n} x^{n} \prod_{j=1}^{N-1} \int_{(0, \infty)} u_{j}^{n} \exp \left(-u_{j}\right) d u_{j}=\sum_{n \geq 0}(-1)^{n}(n !)^{N-1} x^{n} .
$$

What happens if $N=1$ or 2 ? The case $N=1$ is trivial yet interesting,

$$
Z_{1}(x)=\int_{0}^{\infty} \exp (-u-x u) d u=\frac{1}{1+x} .
$$

Expanding the integral with respect to $x$ we obtain $Z_{1}(x)=\sum_{n \geq 0}(-1)^{n} x^{n}$ and for $x=1$ we rediscover the well-known "equality" $\sum_{n \geq 0}(-1)^{n}=\frac{1}{2}$. 


$$
\int_{0}^{\infty} \frac{\exp (-u)}{1+u} d u=0.5963 \ldots
$$

and therefore concluded

$$
\sum_{n \geq 0}(-1)^{n} n !=0.5963 \ldots
$$

a most astonishing equality! In his beautiful book G. H. Hardy [2] discusses in detail this case $N=2$.

Remark 2.1. The constant $\int_{0}^{\infty} \frac{\exp (-u)}{1+u} d u=0.5963 \ldots$ is called the Euler or the EulerGompertz constant (see [3], [1, Section 6.2], and in particular [1, Section 6.2.4] for the name "Gompertz"). Among the numerous results related to this constant we do not resist to write the following continued fraction expansion:

$$
\int_{0}^{\infty} \frac{\exp (-u)}{1+u} d u=\frac{1}{2-\frac{1}{4-\frac{4}{6-\frac{9}{8-\ddots}}}}
$$

This continued fraction expansion is sometimes attributed to Stieltjes, but in [8] Stieltjes indicated that it was studied by Laguerre. We found indeed in [5, p. 154] that Laguerre considered $e$ times the Prym function ${ }^{1} e Q(\alpha)=\int_{1}^{\infty} e^{1-x} x^{\alpha-1} d x$ and obtained as consecutive approximations of $e Q(0)$ the sequence

$$
\frac{4}{7}, \frac{20}{34}, \frac{124}{209}, \frac{920}{2546}, \frac{7940}{13327}, \ldots
$$

which are exactly the values of the first few truncatures of the above continued fraction (also see Laguerre [4, p. 77]). Of course $e Q(0)=\int_{0}^{\infty} \frac{\exp (-u)}{1+u} d u=Z_{2}(1)$ : it would thus be interesting to obtain such nice continued fraction expansions for the quantities $Z_{N}(1)$.

More generally, a formula given by Tannery in [9, p. 1699] or an easy rewriting of a formula given by Laguerre in [4, end of Page 75] reads

$$
e^{x} \int_{x}^{\infty} \frac{e^{-t}}{t} d t=\frac{1}{x+1-\frac{1}{x+3-\frac{4}{x+5-\frac{9}{x+7-\ddots}}}}
$$

But

$$
Z_{2}(x)=\int_{0}^{\infty} \frac{\exp (-u)}{1+x u} d u=\frac{1}{x} \int_{0}^{\infty} \frac{e^{-u}}{\frac{1}{x}+u} d u=\frac{1}{x} e^{1 / x} \int_{1 / x}^{\infty} \frac{e^{-t}}{t} d t
$$

\footnotetext{
${ }^{1}$ Note that there seems to be a misprint in the formula given by Laguerre, where $e^{1-x}$ is replaced by $e^{-x}$, see the original definition by Prym [7, p. 169].
} 
Hence

$$
Z_{2}(x)=\frac{1}{1+x-\frac{x^{2}}{1+3 x-\frac{4 x^{2}}{1+5 x-\frac{9 x^{2}}{1+7 x-\ddots}}}}
$$

\section{The Borel operator}

The sequence $Z_{N}$ can be defined recursively by means of the so-called Borel operator

$$
B: f \mapsto \int_{0}^{\infty} \exp (-u) f(u x) d u
$$

The Borel operator applies the series $\sum_{n \geq 0} f^{(n)}(0) \frac{x^{n}}{n !}$ onto $\sum_{n \geq 0} f^{(n)}(0) x^{n}$.

Using the relation $Z_{0}(x)=\exp (-x)$ and $Z_{N+1}=B Z_{N}$, we see that the integral $Z_{N}$ is therefore the $N$ th iterate $B^{N}$ of $x \mapsto \exp (-x)$, or equivalently the $(N-1)$ st iterate $B^{N-1}$ of $x \mapsto(1+x)^{-1}$.

\section{The Abel-Plana summation and the $\Gamma$ function}

In this section we study the behavior of $Z_{N}$ when $N$ goes to infinity. Note that for real $x \geq 0$, the sequence $N \mapsto Z_{N}(x)$ is bounded from above by 1 and furthermore it is increasing. Indeed let $\Delta_{N}(x)=Z_{N+1}(x)-Z_{N}(x)$ and $\Pi_{N}(x)=x \prod_{j=1}^{N} u_{j}$. Then

$$
\Delta_{N}(x)=\int_{(0, \infty)^{N}} \exp \left(-\sum_{j=1}^{N} u_{j}\right)\left(\frac{1}{1+\Pi_{N}(x)}-\exp \left(-\Pi_{N}(x)\right)\right) \prod_{j=1}^{N} d u_{j} .
$$

Since $\frac{1}{1+t}-\exp (-t) \geq 0, Z_{N+1}(x) \geq Z_{N}(x)$ as claimed. Therefore $Z_{N}(x)$ tends to a limit which we now compute.

Theorem 4.1. For all real $x \geq 0$, we have $\lim _{N \rightarrow \infty} Z_{N}(x)=1$.

Proof. Since the result is trivial for $x=0$, we may assume $x>0$. We note that $Z_{N}(x)$ can be written as a diverging series

$$
Z_{N}(x)=\sum_{n \geq 0}(-1)^{n} f_{N}(n)
$$

where $f_{N}: s \mapsto \Gamma(1+s)^{N-1} x^{s}$ is an analytic function on the half-plane $\Re(s)>-1$.

By blindly applying the Abel-Plana Formula (see [6, III, formula X]) to this series, we get

$$
\begin{aligned}
Z_{N}(x) & =-\int_{-1 / 2-i \infty}^{-1 / 2+i \infty} \frac{\Gamma(1+z)^{N-1} x^{z}}{2 i \sin (\pi z)} d z \\
& =\int_{-\infty}^{+\infty} \frac{\Gamma(1 / 2+i t)^{N-1} x^{-1 / 2+i t}}{2 \cosh (\pi t)} d t
\end{aligned}
$$


or by displacing the integration contour,

$$
\begin{aligned}
Z_{N}(x) & =1-\int_{1 / 2-i \infty}^{1 / 2+i \infty} \frac{\Gamma(1+z)^{N-1} x^{z}}{2 i \sin (\pi z)} d z \\
& =1-\int_{-\infty}^{+\infty} \frac{\Gamma(3 / 2+i t)^{N-1} x^{1 / 2+i t}}{2 \cosh (\pi t)} d t
\end{aligned}
$$

The convergence of the integrals is provided by the fact the $\Gamma$ function decreases like $\exp \left(-\frac{\pi}{2}|z|\right)$ as $z$ goes to $-1 / 2 \pm i \infty$ (resp. $\left.1 / 2 \pm i \infty\right)$, and $\sin (\pi z)$ increases like $\exp (\pi|z|)$.

Strictly speaking, the Abel-Plana Theorem only applies for $N=0$. However, by applying the Borel operator to the right-hand side and interverting the summations by Fubini's Theorem, we find that

$$
F_{N}(x)=\int_{-\infty}^{+\infty} \frac{\Gamma(1 / 2+i t)^{N-1} x^{-1 / 2+i t}}{2 \cosh (\pi t)} d t
$$

satisfies the same recursion as $Z_{N}(x)$. Indeed,

$$
\begin{aligned}
B F_{N}(x) & =\int_{0}^{\infty} \exp (-u) \int_{-\infty}^{+\infty} \frac{\Gamma(1 / 2+i t)^{N-1}(x u)^{-1 / 2+i t}}{2 \cosh (\pi t)} d t d u \\
& =\int_{-\infty}^{+\infty} \frac{\Gamma(1 / 2+i t)^{N-1} x^{-1 / 2+i t}}{2 \cosh (\pi t)} \int_{0}^{\infty} u^{-1 / 2+i t} \exp (-u) d u d t
\end{aligned}
$$

From the identity $\Gamma(1 / 2+i t)=\int_{0}^{\infty} u^{-1 / 2+i t} \exp (-u) d u$, it follows

$$
B F_{N}(x)=\int_{-\infty}^{+\infty} \frac{\Gamma(1 / 2+i t)^{N} x^{-1 / 2+i t}}{2 \cosh (\pi t)} d t=F_{N+1}(x)
$$

therefore $F_{N}=Z_{N}$.

Now since $|\Gamma(3 / 2+i t)| \leq \frac{\sqrt{\pi}}{2}<1$ for all $t \in \mathbb{R}$, the integral (4.2) converges to 0 when $N$ goes to infinity for all real $x \geq 0$, thus we have proved:

$$
\lim _{N \rightarrow \infty} Z_{N}(x)=1
$$

To conclude this section, we note that this formula for $Z_{N}$ involves a single integral which is much more suitable for numerical computations than the original formula involving a multiple integral. Note also that $N$ need not be an integer...

\section{A differential equation}

It might be worthwhile to mention that the function

$$
Z_{N}(x)=\int_{(0, \infty)^{N}} \exp \left(-\left(\sum_{j=1}^{N} u_{j}\right)-x \prod_{j=1}^{N} u_{j}\right) \prod_{j=1}^{N} d u_{j}
$$


is a solution of a differential equation of order $N-1$ with polynomial coefficients.

Indeed, the shortest way to establish this is to introduce the linear operator $U$ defined by $U(z)=(x z)^{\prime}$. Clearly $U\left(x^{n}\right)=(n+1) x^{n}$ so that $U^{k}\left(x^{n}\right)=(n+1)^{k} x^{n}$. Then

$$
\begin{aligned}
U^{N-1} Z_{N}(x) & =U^{N-1} \sum_{n \geq 0}(-1)^{n}(n !)^{N-1} x^{n} \\
& =\sum_{n \geq 0}(-1)^{n}(n !)^{N-1}(n+1)^{N-1} x^{n} \\
& =\sum_{n \geq 0}(-1)^{n}((n+1) !)^{N-1} x^{n} ; \\
x U^{N-1} Z_{N}(x) & =\sum_{n \geq 0}(-1)^{n}((n+1) !)^{N-1} x^{n+1} \\
& =1-Z_{N}(x) .
\end{aligned}
$$

The function $Z_{N}(x)$ is thus solution of the $(N-1)$-st order differential equation

$$
x U^{N-1} y+y=1
$$

with initial conditions

$$
y(0)=1, y^{\prime}(0)=-1, \ldots, y^{(N-2)}(0)=(-1)^{n-2}((N-2) !)^{N-1} .
$$

The reader may well criticize the above proof since it involves divergent series. There is however no problem in justifying the result by applying the operator $U$ to the integral representation of $Z_{N}(x)$; the calculations are just slightly more cumbersome.

Example 5.1. $Z_{2}(x), Z_{3}(x), Z_{4}(x)$ are respectively solution of the equations

$$
\begin{aligned}
x^{2} y^{\prime}+(x+1) y & =1 \\
x^{3} y^{\prime \prime}+3 x^{2} y^{\prime}+(x+1) y & =1 \\
x^{4} y^{\prime \prime \prime}+6 x^{3} y^{\prime \prime}+7 x^{2} y^{\prime}+(x+1) y & =1
\end{aligned}
$$

The reader will recognize the numbers above as the Stirling numbers of the second kind. This can be proved by noting that both families of numbers obey the formula

$$
a_{n+1, k}=k a_{n, k}+a_{n, k-1} .
$$

\section{An unconventional spin system}

We now assume that $\mu$ is the Bernoulli measure on $\{-1,+1\}^{N}$ :

$$
Z_{N}(x)=\frac{1}{2^{N}} \sum_{\underline{u} \in\{ \pm 1\}^{N}} \exp \left(-H\left(\sum_{j=1}^{N} u_{j}\right)-x \prod_{j=1}^{N} u_{j}\right) .
$$

We interpret $Z_{N}$ as the partition function of a certain spin system which we describe below. Conventional spin systems are discussed for example in C. J. Thompson [10].

Imagine an $N$-component particle, each component of which has a spin $u_{j}= \pm 1$, and which are instantaneously influenced by the $N-1$ others. The "total" spin of the particle, 
i.e., its sign is $\prod_{j=1}^{N} u_{j}$. A real external field $H$ acts on the spins. The Hamiltonian attached to the spin system in state $\underline{u}=\left(u_{1}, u_{2}, \ldots, u_{N}\right)$ with external field $-H$ is then given by

$$
x \prod_{j=1}^{N} u_{j}+H \sum_{i=1}^{N} u_{j}
$$

The behavior of the spin system is controlled by the partition function, in particular by its thermodynamical limit

$$
\lim _{N \rightarrow \infty} \frac{\log Z_{N}(x)}{N}
$$

Theorem 6.1. For all real $x \geq 0$,

$$
Z_{N}(x)=\cosh (x) \cosh (H)^{N}-(-1)^{N} \sinh (x) \sinh (H)^{N} .
$$

Proof. By using the relation $\exp (-t)=\cosh (t)-\sinh (t)$, we write

$$
Z_{N}(x)=\frac{1}{2^{N}} \sum_{\underline{u} \in\{ \pm 1\}^{N}} \exp \left(-H\left(\sum_{j=1}^{N} u_{j}\right)\right)\left(\cosh \left(x \prod_{j=1}^{N} u_{j}\right)-\sinh \left(x \prod_{j=1}^{N} u_{j}\right)\right) .
$$

Since $\prod_{j=1}^{N} u_{j}= \pm 1$, cosh is even and sinh is odd, it follows that

$$
Z_{N}(x)=\frac{1}{2^{N}} \sum_{\underline{u} \in\{ \pm 1\}^{N}} \exp \left(-H\left(\sum_{j=1}^{N} u_{j}\right)\right)\left(\cosh (x)-\sinh (x) \prod_{j=1}^{N} u_{j}\right) .
$$

The following two formulas are easily proved by recursion on $N$ :

$$
\begin{aligned}
\sum_{\underline{u} \in\{ \pm 1\}^{N}} \exp \left(H\left(\sum_{j=1}^{N} u_{j}\right)\right) & =(2 \cosh (H))^{N} \\
\sum_{\underline{u} \in\{ \pm 1\}^{N}} \exp \left(H\left(\sum_{j=1}^{N} u_{j}\right)\right) \prod_{j=1}^{N} u_{j} & =(2 \sinh (H))^{N} .
\end{aligned}
$$

From Equation (6.1) it follows:

$$
Z_{N}(x)=\cosh (x) \cosh (H)^{N}-(-1)^{N} \sinh (x) \sinh (H)^{N} .
$$

Remark 6.2. Theorem 6.1 above implies that $Z_{N}(x) \underset{N \rightarrow \infty}{\sim} \cosh (H)^{N} \cosh (x)$, so that

$$
\lim _{N \rightarrow \infty} \frac{\log Z_{N}(x)}{N}=\log \cosh (H)
$$

which happens to be independent of $x$ and which is continuous with respect to $H$. The system has no critical value of the external field and therefore presents no phase transition. 


\section{A disturbed Ising chain}

In the preceding section we described an unconventional spin system. We now turn to the most familiar one, namely the one dimensional Ising chain (see [10]) with Hamiltonian

$$
H \sum_{j=1}^{N} u_{j}+J \sum_{j=1}^{N} u_{j} u_{j+1}
$$

where $J$ is a "coupling constant". Actually this Hamiltonian corresponds to the parameters $-H$ and $-J$ but that makes no essential difference for our computation.

We consider in fact a perturbed Ising chain with the additional term $x \prod_{j=1}^{N} u_{j}$. The Hamiltonian is therefore

$$
\mathcal{H}(\underline{u})=H \sum_{j=1}^{N} u_{j}+J \sum_{j=1}^{N} u_{j} u_{j+1}+x \prod_{j=1}^{N} u_{j}
$$

and the partition function is now

$$
Y_{N}=\frac{1}{2^{N}} \sum_{\underline{u} \in\{ \pm 1\}^{N}} \exp (-\mathcal{H}(\underline{u}))
$$

which we propose to compute where we need to specify $u_{N+1}$. Following most textbooks, we simplify the model by assuming that the chain is cyclic: $u_{N+1}=u_{1}$.

Theorem 7.1. Define

$$
\begin{aligned}
& \lambda_{ \pm}=\exp (-J) \cosh (H) \pm\left(\exp (-2 J) \cosh (H)^{2}+2 \sinh (2 J)\right)^{\frac{1}{2}} \\
& \bar{\lambda}_{ \pm}=\exp (-J) \sinh (H) \pm\left(\exp (-2 J) \sinh (H)^{2}-2 \sinh (2 J)\right)^{\frac{1}{2}}
\end{aligned}
$$

Then

$$
Y_{N}=\frac{1}{2^{N}} \cosh (x)\left(\lambda_{+}^{N}+\lambda_{-}^{N}\right)-\frac{(-1)^{N}}{2^{N}} \sinh (x)\left(\bar{\lambda}_{+}^{N}+\bar{\lambda}_{-}^{N}\right) .
$$

Proof. Observe as in Section 6 that

$$
Y_{N}=\frac{\cosh x}{2^{N}} Y_{N}^{\prime}-\frac{\sinh x}{2^{N}} Y_{N}^{\prime \prime}
$$

where

$$
\begin{aligned}
Y_{N}^{\prime} & =\sum_{\underline{u} \in\{ \pm 1\}^{N}} \exp \left(-H \sum_{j=1}^{N} u_{j}-J \sum_{j=1}^{N} u_{j} u_{j+1}\right), \\
Y_{N}^{\prime \prime} & =\sum_{\underline{u} \in\{ \pm 1\}^{N}} \exp \left(-H \sum_{j=1}^{N} u_{j}-J \sum_{j=1}^{N} u_{j} u_{j+1}\right) \prod_{j=1}^{N} u_{j} .
\end{aligned}
$$

The classical way to compute $Y_{N}^{\prime}$ is to introduce the $2 \times 2$ transfer matrix

$$
L_{1}=\left(\begin{array}{cc}
L_{1}(1,1) & L_{1}(1,-1) \\
L_{1}(-1,1) & L_{1}(-1,-1)
\end{array}\right)
$$


where

$$
L_{1}\left(u_{1}, u_{2}\right)=\exp \left(-\frac{H}{2}\left(u_{1}+u_{2}\right)-J u_{1} u_{2}\right) .
$$

In other words

$$
L_{1}=\left(\begin{array}{cc}
\exp (-H-J) & \exp (J) \\
\exp (J) & \exp (H-J)
\end{array}\right) .
$$

Then

$$
\begin{aligned}
Y_{N}^{\prime} & =\sum_{\underline{u} \in\{ \pm 1\}^{N}} L_{1}\left(u_{1}, u_{2}\right) L_{1}\left(u_{2}, u_{3}\right) \ldots L_{1}\left(u_{N}, u_{1}\right) \\
& =\sum_{u_{1} \in\{ \pm 1\}} L_{1}^{N}\left(u_{1}, u_{1}\right)=\operatorname{Trace}\left(L_{1}^{N}\right)=\lambda_{+}^{N}+\lambda_{-}^{N}
\end{aligned}
$$

where $\lambda_{+}$and $\lambda_{-}$are the eigenvalues of $L_{1}$, i.e., the solutions of

$$
\lambda^{2}-2 \lambda \exp (-J) \cosh (H)+\exp (-2 J)-\exp (2 J)=0 .
$$

Therefore

$$
\lambda_{ \pm}=\exp (-J) \cosh (H) \pm\left(\exp (-2 J) \cosh (H)^{2}+2 \sinh (2 J)\right)^{\frac{1}{2}} .
$$

The computation of $Y_{N}^{\prime \prime}$ is quite similar. Let

$$
L_{2}=\left(\begin{array}{cc}
L_{2}(1,1) & L_{2}(1,-1) \\
L_{2}(-1,1) & L_{2}(-1,-1)
\end{array}\right)
$$

where

$$
L_{2}\left(u_{1}, u_{2}\right)=u_{1} \exp \left(-\frac{H}{2}\left(u_{1}+u_{2}\right)-J u_{1} u_{2}\right)
$$

so that

$$
L_{2}=\left(\begin{array}{cc}
\exp (-H-J) & \exp (J) \\
-\exp (J) & -\exp (H-J)
\end{array}\right)
$$

then

$$
\begin{aligned}
Y_{N}^{\prime \prime} & =\sum_{\underline{u} \in\{ \pm 1\}^{N}} L_{2}\left(u_{1}, u_{2}\right) L_{2}\left(u_{2}, u_{3}\right) \ldots L_{2}\left(u_{N}, u_{1}\right) \\
& =\sum_{u_{1} \in\{ \pm 1\}} L_{2}^{N}\left(u_{1}, u_{1}\right)=\operatorname{Trace}\left(L_{2}^{N}\right)=\bar{\lambda}_{+}^{N}+\bar{\lambda}_{-}^{N}
\end{aligned}
$$

where $\bar{\lambda}_{+}$and $\bar{\lambda}_{-}$are the eigenvalues of $L_{2}$, i.e., the solutions of

$$
\lambda^{2}+2 \lambda \exp (-J) \sinh (H)-\exp (-2 J)+\exp (2 J)=0 .
$$

Therefore

$$
\bar{\lambda}_{ \pm}=-\exp (-J) \sinh (H) \pm\left(\exp (-2 J) \sinh (H)^{2}-2 \sinh (2 J)\right)^{\frac{1}{2}} .
$$

Finally

$$
Y_{N}=\frac{1}{2^{N}} \cosh (x)\left(\lambda_{+}^{N}+\lambda_{-}^{N}\right)-\frac{(-1)^{N}}{2^{N}} \sinh (x)\left(\bar{\lambda}_{+}^{N}+\bar{\lambda}_{-}^{N}\right) .
$$


Remark 7.2. The reader will easily verify that for $J=0$ we obtain the value of $Z_{N}$ computed in Section 6.

Remark 7.3. It is not difficult to see that $\max \left\{\left|\lambda_{-}\right|,\left|\bar{\lambda}_{-}\right|,\left|\bar{\lambda}_{+}\right|\right\}<\lambda_{+}$. Hence $Y_{N} \sim$ $\frac{1}{2^{N}} \cosh (x)\left(\lambda_{+}^{N}\right)$ when $N$ goes to infinity. This implies that the following limit exists, is continuous in both variables $J$ and $H$, and is independent of $x$ (as in Remark 6.2); therefore the system has no phase transition:

$$
\begin{aligned}
\lim _{N \rightarrow \infty} \frac{\log Y_{N}}{N} & =\log \frac{\lambda_{+}}{2} \\
& =\log \left(\frac{(\exp (-J) \cosh (H)}{2}+\frac{\left(\exp (-2 J) \cosh (H)^{2}+2(\sinh (2 J))^{\frac{1}{2}}\right.}{2}\right) .
\end{aligned}
$$

\section{Conclusion and acknowledgements}

This article illustrates a classical fact, namely that one formula may well lead to far distant and unexpected developments. Unifying themes is probably one of the most exciting aspects of mathematics.

We warmly thank H. Cohen and A. Lasjaunias for their very kind help. We are very grateful to J.-Y. Yao and to the referees for their precise and constructive remarks and comments. J.-P. A. was partially supported by the ANR project ANR-12-IS01-0002 "FAN" (Fractals et Numération).

\section{References}

[1] S. R. Finch, Mathematical constants, volume 94 of Encyclopedia of Mathematics and its Applications, Cambridge University Press, Cambridge, 2003, doi:10.1017/CBO9780511550447, http://dx.doi.org/10.1017/CB09780511550447.

[2] G. H. Hardy, Divergent Series, Oxford, at the Clarendon Press, 1949.

[3] J. C. Lagarias, Euler's constant: Euler's work and modern developments, Bull. Amer. Math. Soc. (N.S.) 50 (2013), 527-628, doi:10.1090/S0273-0979-2013-01423-X, http://dx.doi . org/10.1090/S0273-0979-2013-01423-X.

[4] Laguerre, Sur l'intégrale $\int_{x}^{\infty} \frac{e^{-x} d x}{x}$, Bull. Soc. Math. France 7 (1879), 72-81, http: / /www . numdam.org/item? id=BSMF_1879_7 _ 72 _1.

[5] E. Laguerre, Sur la réduction en fractions continues d'une fraction qui satisfait à une équation différentielle linéaire du premier ordre dont les coefficients sont rationnels, J. Math. Pures Appl. 1 (1885), 135-166, http: / / eudml.org/doc/234408.

[6] E. Lindelöf, Le calcul des résidus et ses applications à la théorie des fonctions, GauthierVillars, Imprimeur-Libraire, 1905, http: / / www . gutenberg . org/ebooks / 29781.

[7] F. E. Prym, Zur Theorie der Gammafunction, J. Reine Angew. Math. 82 (1877), 165-172, doi: 10.1515/crll.1877.82.165, http://dx.doi.org/10.1515/crl1.1877.82.165.

[8] T.-J. Stieltjes, Recherches sur les fractions continues, Ann. Fac. Sci. Toulouse Sci. Math. Sci. Phys. 8 (1894), J1-J122, http: / /www. numdam.org/ item? id=AFST_1894_1_8_4_ J1_0.

[9] J. Tannery, Sur les intégrales eulériennes., C. R. Acad. Sci., Paris 94 (1882), 1698-1701.

[10] C. J. Thompson, Mathematical statistical mechanics, The Macmillan Co., New York; CollierMacmillan Ltd., London, 1972, a Series of Books in Applied Mathematics. 